\title{
Fifteen Year Outcome of the Ceraver Hermes Posterior-Stabilized Total Knee Arthroplasty: Safety of the Procedure with Experienced and Inexperienced Surgeons
} \author{
Paolo Filippini ${ }^{3}$ and Alexandre Poignard ${ }^{3}$ \\ ${ }^{1}$ University Paris XII, Hospital Henri Mondor, 94010 Creteil, France \\ ${ }^{2}$ Hospital de Pointe à Pitre Guadeloupe, France \\ ${ }^{3}$ Hospital Henri Mondor, 94010 Creteil, France
}

Philippe Hernigou ${ }^{*}, 1$, Olivier Manicom ${ }^{2}$, Charles Henri Flouzat-Lachaniete ${ }^{3}$, Xavier Roussignol ${ }^{3}$,

\begin{abstract}
We wished to determine whether total knee replacement (TKA) performed by young surgeons increased rates of mortality and complications compared with TKA performed by senior surgeons using the same model of arthroplasty. There were no significant pre-operative differences between the groups in terms of age, gender, height, weight, body mass index, diagnosis, comorbidity and duration of follow-up, which was a mean of 15 years in both groups. Hence, we assessed the 15 year survival of the first 150 Ceraver Posterior-Stabilized total knee arthroplasties undertaken by young surgeons (aged of less than 30 years) in formation in a single university hospital setting (Group B). We used survival curve analysis, with strict definitions regarding end-points, and evaluated a number of different endpoint criteria to assess the outcome and to compare the results with those obtained by the two seniors (aged of more than 40 years) with their 50 first implantations (Group A). The clinical results and survival rate of implants at intermediate to long-term follow-up were similar in both Groups. Kaplan-Meier survival analysis, with revision as the endpoint for failure, showed that the rate of survival at ten years was $96 \%$ (95\% CI, 93 to 100) in both groups. At fifteen years the rate of survival was $91 \%$ ( $95 \%$ CI, 85 to 97 ) in group B, and $92 \%$ (95\% CI, 90 to 94 ) in group A. The implant used in this series appears particularly safe since the usual complications observed with posterior stabilized arthroplasties were not observed even with young surgeons.
\end{abstract}

\section{INTRODUCTION}

From a theoritical point of vue, experience is an important factor in obtaining a satisfactory result in arthroplasty surgery, with surgeons undertaking a lower number of knee replacements per annum having lower scores in their patients than those undertaking greater numbers. However, there are no published data on the outcome of knee arthroplasty undertaken by surgeons at the beginning of their practice coumpared with results obtained by senior surgeons; and the results may change according to the implant, since some implants may have better tolerance with this problem. Hence, we assessed the 15 year survival of the first 150 Ceraver Posterior-Stabilized total knee arthroplasties undertaken by young surgeons (aged of less than 30 years) in formation in a single university hospital setting. We used survival curve analysis, with strict definitions regarding end-points, and evaluated a number of different endpoint criteria to assess the outcome and to compare the results with those obtained by the two seniors (aged of more than 40 years) with their 50 first implantations.

*Address correspondence to this author at the Department of Orthopaedics Surgery, University Paris XII, Hospital Henri Mondor, 94010 Creteil, France; Tel: 33149812601; Fax: 33149812608;

E-mail: philippe.hernigou@wanadoo.fr

\section{MATERIAL AND METHODS}

\section{Implants}

In the early 1990s, several design modifications were made to the original Cloutier bicruciate arthroplasty $[1,2]$ to obtain a Posterior-Stabilized Condylar prosthesis. Finite element analysis of total knee replacements at this time had showed the enhanced load transmission to the underlying bone with the addition of metal-backing to the tibial component. This feature present on the Cloutier arthroplasty was incorporated into the design. The design of the posterior stabilized Ceraver Hermes TKA incorporate a femoral cam mechanism that articulates with a tibial post to act as a functional substitute for the PCL (Fig. 1). Also, early clinical experience with the original design of the first Insall psterior stabilized knees suggested some concern for the patellofemoral articulation. To address these concerns, changes were made to the femoral component to allow for a smoother patellofemoral transition. Additionally, a wider variety of prosthesis sizes (five different sizes) was introduced to allow better prosthetic fit for individual patients.

\section{Patients}

Between 1990 and 1995, 195 patients underwent 250 total knee arthroplasties in the same orthopaedic department 
of the hospital by two senior surgeons (100 TKA; Group A) and 12 younger surgeons (150 TKA; Group B). Prior to these 150 knee replacements, these younger surgeons had undertaken five knee replacements with assistance. The procedures were undertaken consecutively and the implant was always the same: the posterior stabilized Ceraver TKA.

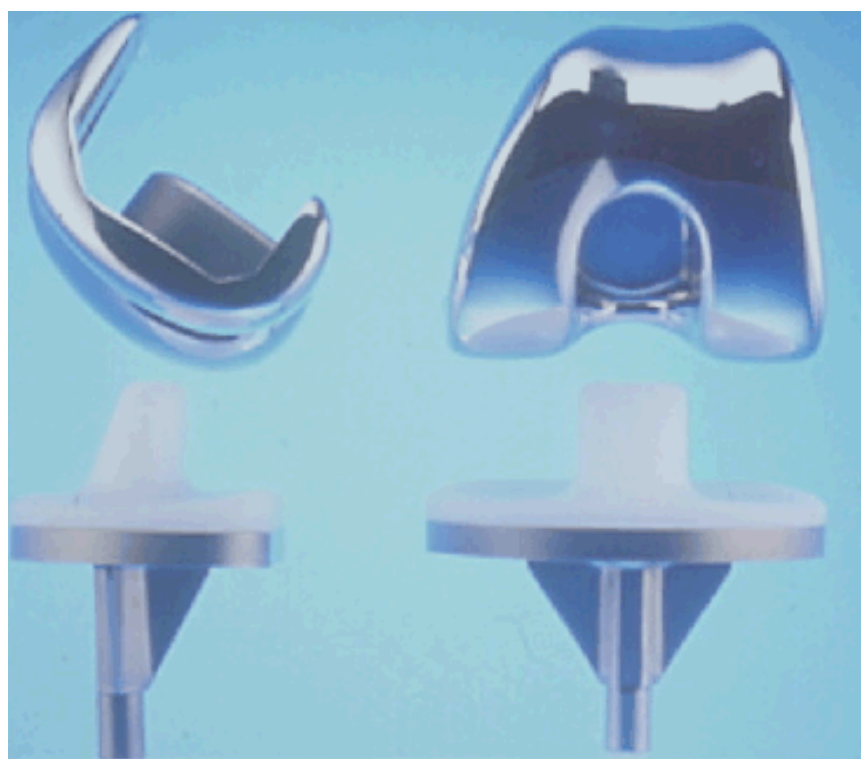

Fig. (1). Photography of the implant.

There were 59 males and 136 females with an average age of 73 years (Range 46-88 Years) at the time of surgery. These patients had osteoarthritis.

There were no significant pre-operative differences between the groups in terms of age, gender, height, weight, body mass index, diagnosis, comorbidity and duration of follow-up, which was a mean of 15.2 years in the Group A and 15.4 years in the Group B.

All procedures were undertaken with routine prophylactic antibiotics, tourniquet and the use of a medial parapatellar arthrotomy except in 25 genu valgum. The patella was resurfaced with the exception of one patient who had had a previous patellectomy. The post-operative regimen included anticoagulation for thromboprophylaxis and physiotherapy-led flexion exercices.

\section{Surgical Technique}

The surgical principles for implanting a fixed bearing, posterior cruciate ligament-substituting design have been well-documented and have their roots in the technique originally used by Insall with the Total Condylar Knee prosthesis where both cruciate ligaments were sacrificed. The basic objective was to produce a knee with symmetric, rectangular flexion and extension spaces with equal soft tissue tension [3]. Instruments were used to restore the mechanical axis to the knee, size and place the components in the proper axial and rotary positions, and resurface the patella. The correction of the deformity was achieved with soft tissue releases, and precise soft tissue balancing in flexion and extension. Both cruciate ligaments were excised in the surgical technique.

A full range of implant sizes were used, with the most common being femoral size 3 , tibial size 3 and patella size
36. Ninety-four knees required an 7-mm insert, which was the standard sized insert at the time. Seventy-two required a $9 \mathrm{~mm}$ insert, and the rest were equally divided between 11 $\mathrm{mm}$ and $13 \mathrm{~mm}$ inserts.

\section{Assessment}

Patients were examined at 3 months, 1, 2, 5, 7, 10, 15, and 19 years (for the patients with the most important follow-up), with a standard weight-bearing antero-posterior and lateral X-Ray at each clinic attendance. The clinical follow-up was by a clinical fellow who was not a member of the surgical team and had no knowledge of the radiological findings. Pre- and postoperative assessments were made according to the system of the Knee Society and of the Hospital for Special Surgery (HSS). Pre- and post-operative radiographs including anteroposterior (AP) and skyline views were assessed for limb alignment, position of the implant and presence and location of any radiolucent lines at the cement-bone interface according to the recommendation of the Knee Society.

The preoperative and postoperative deformities was measured on weight-bearing radiographs of the whole limb (hip-knee-ankle angle); all the subjects were asked to stand with the anterior part of the knees facing forward and the posterior part of the knees facing the film. A cassette holding long radiographs was placed behind the patient. Both lower extremities were included in one radiograph. The weightbearing anteroposterior radiograph of the entire lower limb was made with the X-Ray beam centered at the knees at a distance of three meters and the foot orientated in the direction of the X-Ray beam. The hip-knee-ankle angle was formed by the angle between the line joining the center of the femoral head to the center of the knee and the line joining the center of the knee to the center of the ankle. Normally these axes form a straight line (180 degrees). Therefore in presence of a varus deformity the angle is less than 180 degrees.

\section{Statistical Analysis}

This was undertaken using SPSS for Windows version 10 (SPSS Inc., Chicago, Illinois, USA) with the independent sample t-test used for continuous variables and the chisquared and Fisher's exact tests for dichotomous variables. Mortality and morbidity rates between males and females were compared using logistic regression analysis with the level of significance set at 5.0\% ( $<=0.05)$. The KaplanMeier survivorship was used in the analysis of the failurefree rate.

\section{RESULTS}

Complete survival data were reviewed for all 250 knees with an average theoritical follow-up of 17 years after implantation. 47 patients had died before reaching 10 years. None died as a direct result of their surgery. The mean radiological follow-up was 13 years, as 25 patients were unable to attend for X-Ray due to incapacity or other on-going medical problems. These patients were contacted and interviewed by telephone. As a result the mean clinical and radiological follow up of the series was 15 years. 
The peri-operative (until three months after operation) mortality rate of patients of Group B was similar to that of patients of Group A. One of 150 patients $(0.6 \%)$ of Group B and one of 100 patients (1\%) of Group A died from myocardial infarction within two and three weeks of the operation, respectively. This difference was not significant (chi-squared test, $\mathrm{p}=0.738$ ).

Blood transfusion was given to those whose haemoglobin level fell below $8 \mathrm{~g} / \mathrm{dl}$ post-operatively. Patients in Group B had more transfusions than those in the Group A (3.6 units, SD $1.1 v s 1.5$ units, SD 0.5) (Student's t-test, $\mathrm{p}<0.0001$ ). Patients in Group B also had a greater amount (paired t-test $p$ $<0.001)$ of peri-operative fluid replacement than those in the Group A. There was no reported case of pulmonary embolism as a complication of deep-vein thrombosis or fat embolism. The morbidity rate in males was lower than that in females, but this difference was not significant (logistic regression analysis, $\mathrm{p}=0.778$ ).

\section{Clinical Data}

The clinical results and survival rate of implants at intermediate to long-term follow-up were similar in both Groups. The mean pre-operative Knee Society knee and functional scores were 37.5 points (16 to 53 ) and 15 points ( 0 to 20 ) respectively in the Group B, and 36.0 points (10 to 58 ) and 17 points ( 0 to 30$)$ respectively in the Group A. The differences were not significant for either the knee (Student's t-test, $\mathrm{p}=0.175$ ) or functional scores (Student's t-test, $\mathrm{p}=$ $0.125)$. The mean pre-operative HSS knee score was 39.4 points (6 to 58 ) in the Group B and 42.5 points (15 to 55$)$, in the Group A. This difference was not significant (Student's ttest, $p=0.113$ ). The mean Knee society knee and functional scores at final follow-up were 91 points (42 to 100) and 82 points (25 to 100) respectively in the Group B, and 89 points (58 to 100 ) and 84 points (35 to 100) respectively in the Group A. These differences were not significant for either of the knee scores (Student's t-test, $\mathrm{p}=0.136$ ) or functional scores (Student's t-test, $\mathrm{p}=0.145$ ). The mean HSS knee score at final follow-up was 92.4 points (45 to 100) in the Group B and 90.2 points (37 to 100) in Group A group. This difference was not significant (Student's t-test, $\mathrm{p}=0.087$ ).

\section{Radiological Data}

Patients who had Group B achieved the same quality of radiological results as those undergoing Group A. The alignment of components and the incidence of radiolucent lines were similar in both groups. There were no significant differences in revision rates in Group B or Group A (chisquared and Fisher's exact test $\mathrm{p}=0.735$ ). In the Group B group, 1 knee $(0.6 \%)$ was revised for osteolysis, $1(0.6 \%)$ deep infection, $1(0.6 \%)$ for aseptic loosening, and $1(0.6 \%)$ for supracondylar fracture. In the Group A, 1 knee (1\%) was revised for deep infection, none for osteolysis and $2(2 \%)$ for aseptic loosening, and one for dislocation. Kaplan-Meier survival analysis, with revision as the endpoint for failure, showed that the rate of survival at ten years was $96 \%(95 \%$ CI, 93 to 100) in both groups. At fifteen years the rate of survival was $91 \%(95 \% \mathrm{CI}, 85$ to 97$)$ in group B, and $92 \%$ ( $95 \%$ CI, 90 to 94 ) in group A.

\section{DISCUSSION}

Surgeons embark on a learning curve when undertaking a new procedure that varies amongst surgeons and the procedure that they are undertaking. There is possibility that trainee surgeons may have poorer results and increased complications than senior surgeons more familier with the operative technique. However, no series has measured the radiological positioning of total knee arthroplasties and found significant differences between consultants and trainees, so the argument is not clear cut. Although the medium- and long-term results of knee arthroplasty are well published, details of survivorship in knee arthroplasty for surgeons at the beginnig or at the stars of their practice are not evaluated.

The 10-year survival for total knee arthroplasty, using revision surgery as an endpoint, is over $92 \%$ for a number of well-established prostheses [4-6]. The overall 10-year survival in this cohort is $94.96 \%$, which is in fine with the published data [4-6]. It is encouraging that surgeons in the early stages of their careers can achieve such results; however, there are a number of factors that determine the success of a prosthesis, including the surgeon's skill as well as the implant design. Therefore, it cannot be concluded that all surgeons at this stage would achieve the same results with a different prosthesis. What is difficult to assess is how the 'learning curve' of one implant affects the 'learning curve' for a different implant.

The implant used in this series appears particularly safe since the usual complications (wear, clunk syndrome, dislocation) observed with posterior stabilized arthroplasties [7-11] were not observed even with young surgeons. The variability in wear patterns observed among designs may be due to differences in cam-post mechanics, post location, and post geometry. Manufacturers should be motivated to produce implants that maintain the function of the tibial postcam articulation while limiting wear. No wear was observed on the polyethylene of the posts in retrived implants of this series. One of the disadvantages of posterior stabilized TKA include the potential for dislocation in flexion as the tibial post rides underneath the femoral cam. The incidence of dislocation with posterior stabilized TKA is very rare with modern designs $(0.2 \%)$. To prevent knee dislocation it is mandatory that the surgeon balance the knee both in flexion and extension. This complication was observed in only one knee in this series.

There are weaknesses in this paper. We have only presented survival analysis with only one implant. Our study has limitations. It was not randomised and all the patients in the series received cemented TKA in a large-volume centre specialising in joint reconstruction. This may limit the applicability of the findings to other centres.

\section{REFERENCES}

[1] Cloutier JM. Results of the total knee arthroplasty with a nonconstrained prosthesis. J Bone Joint Surg 1983; 65(7): 906-19.

[2] Cloutier JM. Long-term results after nonconstrained total knee arthroplasty. Clin Orthop 1991; 273: 63-5. 
[3] Swanik CB, Lephart SM, Rubash HE. Proprioception, kinesthesia, and balance after total knee arthroplasty with cruciate-retaining and posterior stabilized prostheses. J Bone Joint Surg Am 2004; 86-A: 328-34.

[4] Ranawat CS, Komistek RD, Rodriguez JA, et al. In vivo kinematics for fixed and mobile-bearing posterior stabilized knee prostheses. Clin Orthop Relat Res 2004; 418: 184-90.

[5] Jacobs WC, Clement DJ, Wymenga AB. Retention versus removal of the posterior cruciate ligament in total knee replacement: a systematic literature review within the Cochrane framework. Acta Orthop 2005; 76: 757-68.

[6] Rasquinha VJ, Ranawat CS, Cervieri CL, Rodriguez JA. The pressfit condylar modular total knee system with a posterior cruciatesubstituting design. A concise follow-up of a previous report. J Bone Joint Surg Am 2006; 88-A: 1006-10.
[7] Kilgus DJ, Moreland JR, Finerman GA, et al. Catastrophic wear of tibial polyethylene inserts. Clin Orthop Relat Res 1991; 273: 22331

[8] Puloski SK, McCalden RW, MacDonald SJ, et al. Tibial post wear in posterior stabilized total knee arthroplasty. An unrecognized source of polyethylene debris. J Bone Joint Surg Am 2001; 83-A: 390-7.

[9] Colizza WA, Insall JN, Scuderi GR. The posterior stabilized total knee prosthesis: Assessment of polyethylene damage and osteolysis after a 10-year minimum follow-up. J Bone Joint Surg Am 1995; 77-A: 1713-20.

[10] Beight JL, Yao B, Hozack WJ, et al. The patellar 'clunk' syndrome after posterior stabilized total knee arthroplasty. Clin Orthop Relat Res 1994; 299: 139-42.

[11] Lombardi AV Jr, Mallory TH, Vaughn BK, et al. Dislocation following primary posterior-stabilized total knee arthroplasty. J Arthroplasty 1993; 8: 633-9.

(C) Hernigou et al.; Licensee Bentham Open.

This is an open access article licensed under the terms of the Creative Commons Attribution Non-Commercial License (http://creativecommons.org/licenses/by$\mathrm{nc} / 3.0 /$ ) which permits unrestricted, non-commercial use, distribution and reproduction in any medium, provided the work is properly cited. 